\title{
Experimental results from magnetized-jet experiments executed at the Jupiter Laser Facility
}

\author{
M. J.-E. Manuel ${ }^{1, *}$, C. C. Kuranz, A. M. Rasmus, S. Klein, M. J. MacDonald, M. R. Trantham, J. R. Fein, \\ P. X. Belancourt, R. P. Young, P. A. Keiter, R. P. Drake \\ Department of Atmospheric, Oceanic and Space Sciences, University of Michigan, Ann Arbor, MI \\ B. B. Pollock, J. Park, A. U. Hazi, G. J. Williams, H. Chen \\ Lawrence Livermore National Laboratories, Livermore, CA
}

\begin{abstract}
Recent experiments at the Jupiter Laser Facility investigated magnetization effects on collimated plasma jets. Laserirradiated plastic-cone-targets produced collimated, millimeter-scale plasma flows as indicated by optical interferometry. Proton radiography of these jets showed no indication of strong, self-generated magnetic fields, suggesting a dominantly hydrodynamic collimating mechanism. Targets were placed in a custom-designed solenoid capable of generating field strengths up to 5 Tesla. Proton radiographs of the well-characterized B-field, without a plasma jet, suggested an external source of trapped electrons that affects proton trajectories. The background magnetic field was aligned with the jet propagation direction, as is the case in many astrophysical systems. Optical interferometry showed that magnetization of the plasma results in disruption of the collimated flow and instead produces a hollow cavity. This result is a topic of ongoing investigation.
\end{abstract}

Keywords: plasma jets, magnetized plasma, laboratory astrophysics

PACS: 52.30.-q, 52.25.Xz, 52.30.Cv,

\section{Introduction}

Magnetized plasma jets are ubiquitous in the universe and found in many classes of astrophysical objects [1, 2]. Often these jets are found as outflows from accretion systems at stellar [3] and galactic [4] scales. The typical magnetohydrodynamic (MHD) model in collimated astrophysical systems relies on shearing the poloidal field $\left(\mathbf{B}_{p o l}=\right.$ $\left.B_{r} \hat{\mathbf{r}}+B_{z} \hat{\mathbf{z}}\right)$ that penetrates the accretion disc. In this geometry, the disc is toroidal $(\theta)$ and its axis is aligned in the z-direction. The sheared poloidal field generates a toroidal component $\left(B_{\theta}\right)$ which further collimates the flow [5] along the $\mathrm{z}$ axis. The collimation of the jet is related to the Lorentz force component $\left(F_{\perp}\right)$ perpendicular to the poloidal field,

$$
F_{\perp}=-\frac{B_{\theta}}{\mu_{0} r} \nabla_{\perp}\left(r B_{\theta}\right)+j_{\theta} B_{p o l},
$$

where $j_{\theta}$ is the toroidal component of the current, and $r$ is the distance from the symmetry axis in SI MKS units. The first term in Eq. 1 describes self-collimation of the jet due

\footnotetext{
* Corresponding author

Email address: mmanuel@umich.edu (M. J.-E. Manuel)

${ }^{1}$ as an Einstein Fellow with the Harvard Smithsonian Astrophysical Observatory
}

to the varying toroidal field and the last term describes magnetic pinching effects. The latter term is studied in this work. These astrophysical systems may be well represented by the Euler MHD equations and scaled [6] to laboratory experiments.

In recent years, multiple platforms have been developed at various laser facilities to create astrophysically relevant plasma jets. Direct irradiation of planar foils has been shown to create plasma jets [7] that may be magnetized by a strong B-field [5]. Also, laser-irradiated hollow cones have been used to hydrodynamically create collimated plasma jets [8, 9]. Results from magnetized-jet experiments executed at the Jupiter Laser Facility (JLF) at the Lawrence Livermore National Laboratory (LLNL) using hollow cone targets are reported herein.

An outline of this paper is as follows. Section 2 describes the targets used in these experiments and the diagnostics implemented to characterize the plasma jet. Field measurements from the pulse-powered solenoid, designed and built at the University of Michigan, are described in Section 3. Measurements taken of unmagnetized jets are shown in Section 4 and optical data taken of magnetized jets are discussed in Section 5. The results of these experiments are summarized in Section 6 and this paper concludes in Section 7 where future projects derived from this work are presented. 


\section{Experimental Setup}

\subsection{Targets}

Irradiated, plastic cones generated collimated plasma jets [8] on the Titan laser. Targets were driven by a 10ns square pulse, with typical rise and fall times of $\sim 10 \%$ and $\sim 20 \%$ of the pulse duration, respectively. Each pulse contained $\sim 300 \mathrm{~J}$ of $2-\omega$ light, of wavelength $\lambda=0.527 \mu \mathrm{m}$. Distributed phase plates shaped the beam to a super gaussian profile with a $\sim 600-\mu$ m diameter. The laser spot was centered on the apex of the cone, as illustrated in Figure 1a, to drive a shock through the cone. When breakout occurs on the backside of the $\sim 90$ - $\mu$ m-thick target, material is accelerated normal to the back surface. These targets were fabricated with a $80^{\circ}$ cone-half-angle, thereby directing the plasma to the central cone axis. As plasma accumulates on-axis, it is further heated and directed outwards, producing a mm-scale, collimated plasma-jet.

Targets were produced using 3D-printing facilities available at the University of Michigan due to the large number of targets required for these experiments. Multiple iterations were performed to optimize the printer settings for these targets. To eliminate part-to-part variation due to environmental effects, all targets were made at the same vertical location and were produced during the same run. Small volumes of 3D-printed plastic were analyzed and found to have a density of $\rho_{3 D} \approx 1.2 \mathrm{~g} / \mathrm{cm}^{3}$ and an atomic composition of $\mathrm{C}_{5} \mathrm{H}_{8} \mathrm{O}_{2} \mathrm{Sb}_{0.03}$, similar to that of standard acrylic. Cones were scanned using a Micro Computerized Tomography (MCT) facility at the University of Michigan and image stacks were processed using ImageJ [10, 11]. Figure 1b illustrates two orthogonal cross-sections derived from the MCT scans. Cones were found to be symmetric in the horizontal $(\mathrm{x})$ direction and slightly asymmetric in the vertical $(\mathrm{y})$ direction due to gravitational effects [12] during the vertical printing process. Small asymmetries

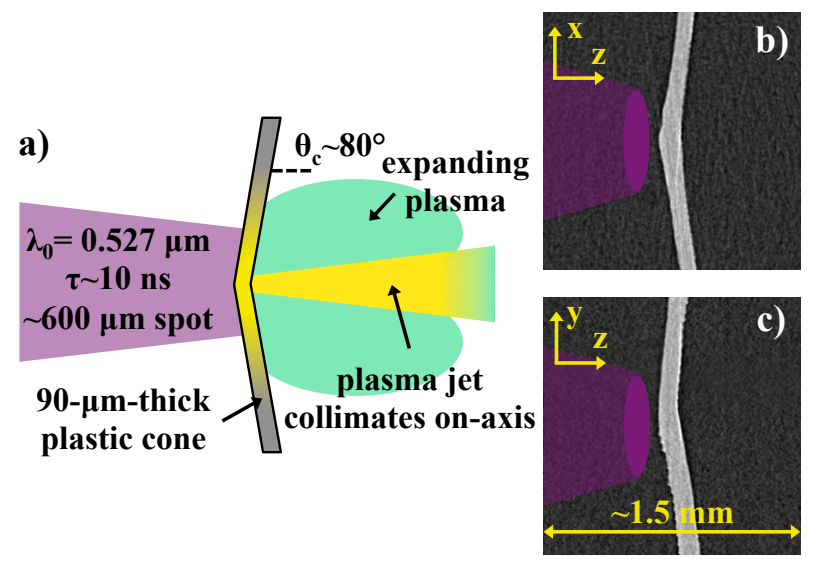

Figure 1: a) Schematic of cone targets irradiated by a 10-ns laser drive. When shocks break out on the backside, material accelerates to the central axis, generating a dense, collimated jet surrounded by background expanding plasma. Orthogonal cross-sections taken at the cone apex of an un-driven, 3D-printed target in the b) horizontal and c) vertical directions. A scaled laser-spot is shown for reference.
$(<10 \mu \mathrm{m})$ were not detrimental to the formation of the mm-scale jets these targets were designed to create.

\subsection{Diagnostic Configuration}

Plasma jets were created in the gap of a solenoid as schematically shown in Figure 2a. Plastic cones were placed in the gap against the edge of the coil housing such that the jet could propagate $\sim 4-\mathrm{mm}$ before crossing into the coil on the opposite side. The long-pulse laser was aligned along the solenoid axis as shown in Figure 2a to create jets parallel to the B-field. An optical probe $\left(\lambda_{p}=0.532-\mu \mathrm{m}\right)$ and short-pulse, proton radiography [13] imaged the plasma in orthogonal directions. The pointing of the Titan shortpulse was orthogonal to the long-pulse on the same horizontal plane. It irradiated a gold-coated silicon wafer [14] to generate protons by target-normal sheath acceleration (TNSA) that were recorded on a filtered radiochromic film (RCF) stack. The film stack was attached to an imaging proton spectrometer [15] that measured the proton spectrum [16] on every shot.

The optical probe beam had a $\sim 12$-ns pulse containing $\sim 2 \mathrm{~mJ}$ of energy. It propagated under the solenoid to a beam splitter beneath the target to vertically probe the plasma jet. A mirror above the target redirected the beam to a splitter, sending half of the beam intensity to a shadowography/Schlieren arm, and half to recombine with the reference beam of the interferometry system, as shown schematically in Figure 2b. The shadowography diagnostic could be switched to Schlieren by inserting a knife edge at the focal plane of that optical path.
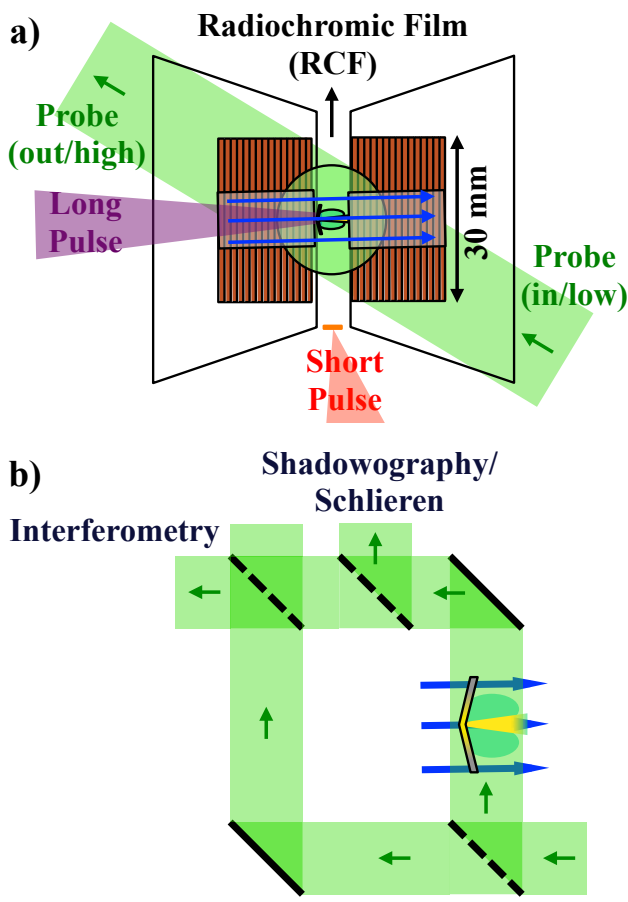

Figure 2: a) Top-down schematic of the plasma jet in the solenoid gap with the laser configuration illustrated. b) Side-view schematic of the optical path for the probe beam. 


\section{Solenoid Implementation}

\subsection{Design Parameters}

A custom-built solenoid delivered axial magnetic field strengths of up to $\sim 5 \mathrm{~T}$ to magnetize laser-generated plasma jets. Details of the manufacturing techniques employed to construct the compact solenoid is discussed by Klein et al. [17], but the design and field characterization is discussed herein. The following formulae were instrumental in determining the final geometry of the system. The vector components of the B-field, as a function of $r$ and $z$, from a single ideal coil carrying a current $I$ with $N$ loops, length $L$, and inner radius $a$ may be written [18] as

$$
\begin{gathered}
B_{r}=\frac{\mu_{0} N I}{2 \pi L}\left[\frac{a r k_{+}^{2}-2}{r k_{+}} K\left(a r k_{+}^{2}\right)+\frac{2}{r k_{+}} E\left(a r k_{+}^{2}\right)-\frac{a r k_{-}^{2}-2}{r k_{-}} K\left(a r k_{-}^{2}\right)-\frac{2}{r k_{-}} E\left(a r k_{-}^{2}\right)\right] \\
B_{z}=\frac{\mu_{0} N I}{4 \pi L}\left[k_{+}\left(z+\frac{L}{2}\right)\left(K\left(a r k_{+}^{2}\right)+\frac{a-r}{a+r} \Pi\left(h^{2}, a r k_{+}^{2}\right)\right)-k_{-}\left(z-\frac{L}{2}\right)\left(K\left(a r k_{-}^{2}\right)+\frac{a-r}{a+r} \Pi\left(h^{2}, a r k_{-}^{2}\right)\right)\right] .
\end{gathered}
$$

Equations 2 and 3 include the functions $K(m), E(m)$, and $\Pi(n, m)$ which are complete elliptic integrals of the first, second, and third kind respectively. The other characteristic parameters are defined as

$$
\begin{gathered}
k_{ \pm}(r, z)=\sqrt{\frac{4}{(a+r)^{2}+(z \pm L / 2)^{2}}}, \\
h(r)=\sqrt{\frac{4 a r}{(a+r)^{2}}},
\end{gathered}
$$

which completes the field description for a single coil. The ideal solenoid can be approximated as the superposition of two identical coils separated by a gap distance $d$ and the total B-field components may be finally expressed as

$$
B_{r, z}^{s o l}=B_{r, z}\left(r, z+\frac{d}{2}+\frac{L}{2}\right)+B_{r, z}\left(r, z-\frac{d}{2}-\frac{L}{2}\right),
$$

where the origin of the coordinate system has been set to the center of the solenoid gap.

Using the constraints given by the experimental geometry and the preceding equations, an optimum design was attained. The solenoid geometry was dictated by requiring that the spatial distribution of the B-field in the gap vary by $\lesssim 5 \%$ and that sufficient access for diagnostics was available. The proton backlighter could not be placed too far inside the solenoid, so the maximum radial size of the coil and housing was fixed by the optical geometry required for proton radiography. The ratio of the inner radius and length of the coil was fixed by the $\mathrm{f} / 7.5$ final optic on the long-pulse beam. To maintain structural integrity, the peak field in the coil was limited to the critical value of $\sim 13 \mathrm{~T}$, the field strength where the magnetic pressure is equal to the approximate yield stress of copper, $\sim 70 \mathrm{MPa}$. These technical specifications led to a Bitter-magnet [19] design based on previous work by Pollock et al. [20].

Figure 3 illustrates the solenoid point-design with the following parameters: $a=4.95 \mathrm{~mm}, d=7 \mathrm{~mm}, L=16.5 \mathrm{~mm}$, $N=15$. B-field variation along the central axis of the solenoid is shown in Figure 3a. The peak field in the coils is $\sim 2.3$ times higher than that of the field attained at the gap center. This implies that for a gap field of $\sim 5 \mathrm{~T}$, the peak field in the coil will be $\sim 11.5 \mathrm{~T}$, lower than the critical $\sim 13 \mathrm{~T}$ limit. The solenoid coils will have additional support to maintain structural integrity, but this design ensures that a gap-field of $5 \mathrm{~T}$ does not push the capabilities of the system. As the coil separation distance $d$ is increased, the gap field becomes more nonuniform. To balance field uniformity in the gap and allow for diagnostic and target access in the center, a coil separation of $7 \mathrm{~mm}$ was chosen. Figure $3 \mathrm{~b}$ illustrates axial field contours, separated by $0.5 \mathrm{~T}$,
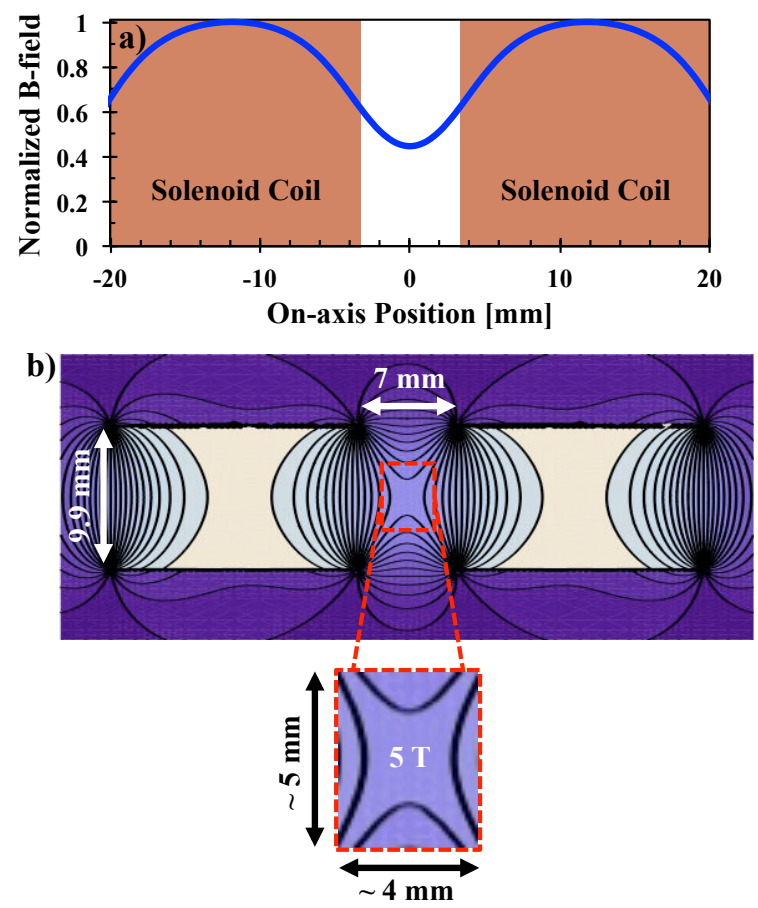

Figure 3: a) Analytic B-field strength on-axis as a function of distance from the center is normalized to the peak field. The envelope of each of the coils is shown. b) Axial field contours calculated from Equations 2-6 with the point-design parameters. An expanded view of the uniform region used to magnetize the jets is also shown. 
a)

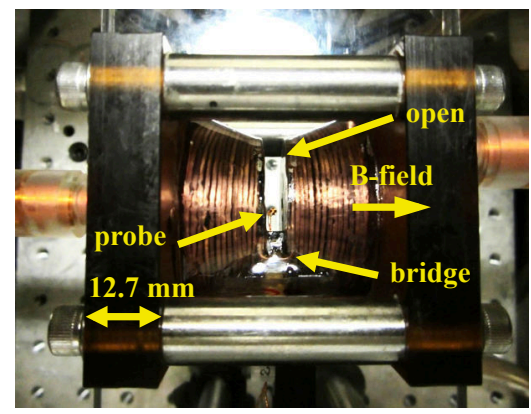

b)

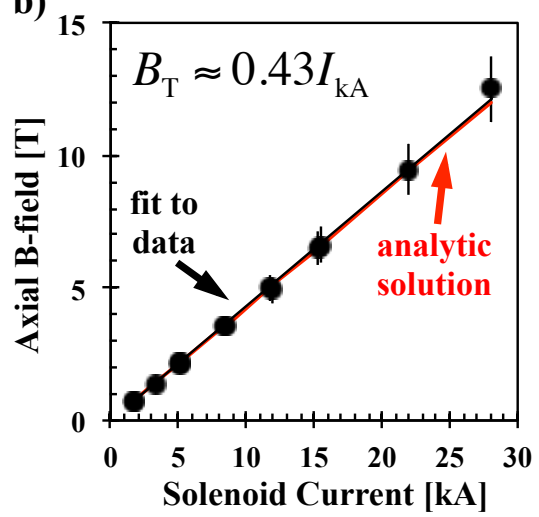

c)

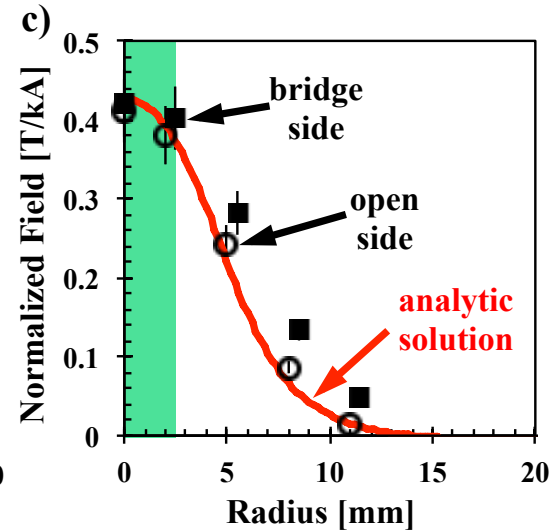

Figure 4: a) Image of the solenoid in the test chamber with the b-dot probe placed at the center. The bridge connecting the two coils across the gap is labeled, as is the 'open' side without the bridge. b) The central, axial B-field is shown to be linear with the applied current. c) B-field measurements demonstrate radially decreasing field strength. The analytic solution is accurate to within $\sim 2 \%$ of the open side measurements, whereas the bridge-side fields are altered at radii $\gtrsim 5 \mathrm{~mm}$ by the presence of the bridge located $\sim 20$ mm from the axis.

as calculated from Equations 2-6. The expanded view of the gap field shows a $\sim 80-\mathrm{mm}^{3}$ cylindrical volume where the field varies by less than $5 \%$ and where the plasma jets were created.

\subsection{Magnetic Probe Measurements}

Figure 4a shows a top-down view of the solenoid with the power cables connected on either side. It was fielded using the pulse-power system [20] at the JLF and characterized in a test chamber at air. The solenoid had an inductance of $\sim 3 \mu \mathrm{H}$ making the system underdamped with a rise time of $\sim 40 \mu$ s and a peak width of $\sim 10 \mu$ s where the field is within $\sim 3 \%$ of the maximum value.

The solenoid design was experimentally verified by measuring the field strength at different locations within the gap. A multi-loop, "b-dot" magnetic probe [17] was constructed with a $\sim 1-\mathrm{mm}^{3}$ tip to map out the spatial variation of the magnetic field. The changing magnetic flux through the wire loops induces a potential in the wire that is measured using an oscilloscope. The B-field normal to

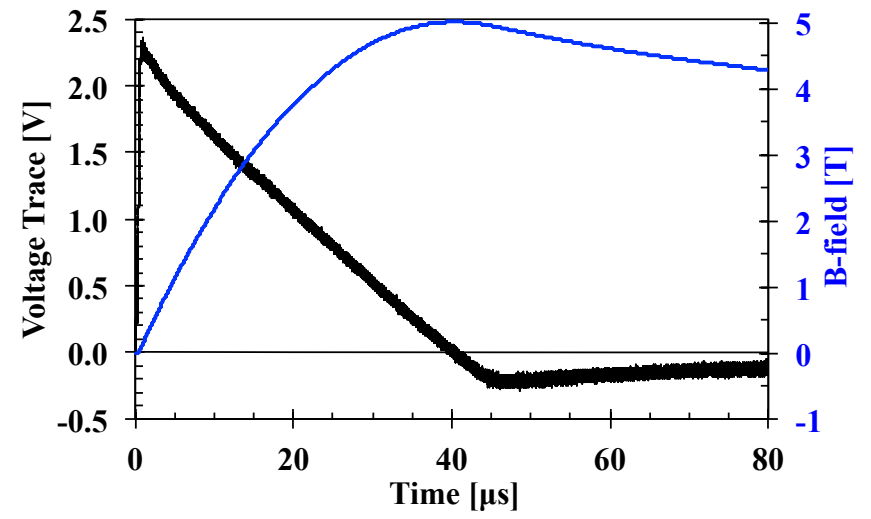

Figure 5: Sample voltage trace (thick) from a $3.5 \mathrm{kV}$ charge and the corresponding $\mathrm{B}(\mathrm{t})$ measurement (thin). the loops is proportional to the time integrated voltage signal $V(t)$,

$$
B_{\text {norm }}(t)=\frac{1}{A_{\text {eff }}} \int_{t_{\text {trig }}}^{t} V\left(t^{\prime}\right) d t^{\prime},
$$

where $t_{\text {trig }}$ is the trigger of the trace and $A_{\text {eff }}$ is the effective area of the wire loops. The probe was calibrated by a known B-field source at the LLNL and found to have $A_{\text {eff }}=8.5 \mathrm{~mm}^{2}( \pm 10 \%)$. Since these probes were not being exposed to any plasma, no additional shielding or circuitry was implemented. A sample b-dot trace and corresponding integral is shown in Figure 5.

Results from axial field measurements taken at the gap center are shown in Figure $4 \mathrm{~b}$ and demonstrate the expected linearity of the system up to currents of $\sim 28 \mathrm{kA} ;{ }^{2}$ note that the $5 \mathrm{~T}$ point design is achieved with a current of $\sim 12 \mathrm{kA}$. The pulsed-power system has the capability to hold a $20 \mathrm{kV}$ charge, though a $3.5 \mathrm{kV}$ charge was sufficient to reach the $5 \mathrm{~T}$ field in the gap. Measurements were also taken at multiple radial locations to map out variation throughout the solenoid gap and explore the effects of the bridge on the axial field. Figure 4c illustrates that the analytic solution represents the radial field variation well on the open side of the solenoid. The bridge causes a measurable effect at radii $\gtrsim 5 \mathrm{~mm}$, but the field is within $\sim 5 \%$ of the maximum up to radial distances of $\sim 2.5 \mathrm{~mm}$ in all directions.

\subsection{Proton Radiography of a Known B-field}

Proton radiography is a diagnostic technique that has been used in many experiments $[13,21,22]$ to probe electromagnetic fields in laser-produced plasmas. Electromagnetic fields will alter an otherwise uniform flux of protons

\footnotetext{
${ }^{2}$ Magnetic forces on the coils during the $\sim 28 \mathrm{kA}$ pulse broke the welds to the bridge resulting in an arc across the gap that broke the structure in multiple locations.
} 
by means of the Lorentz force; thus encoding information about the field structure in modulations of the proton fluence. To extract a quantitative inference of the pathintegrated field strength from proton deflections, some knowledge of the field geometry must be assumed. Monoenergetic proton radiography [23], using exploding-pusher backlighters, has been used to infer electromagnetic field strengths from a 'simple' Z-pinch-like geometry [24], though in this case the current and potentials associated with the system were also inferred from the data. To our knowledge, no proton radiographs, using TNSA or monoenergetic protons, have ever been taken of a known magnetic field distribution.

In these experiments, TNSA protons from gold-coated silicon-wafer targets imaged the solenoid B-field. The first set of proton radiographs in Figure 6a demonstrate the nearly flat spatial distributions of proton fluence at energies of $\sim 2$ and $\sim 7 \mathrm{MeV}$. The second set of images in Figure 6a show the results from radiographing the gap field with an on-axis magnitude of $\sim 2 \mathrm{~T} .{ }^{3}$ The centerline (CL) illustrates the location of the backlighter and the axis of the solenoid. The field direction is left-to-right, as indicated, and should deflect all protons downward in this view. However, a strong focusing of $\sim 2-\mathrm{MeV}$ protons is clearly observed with a net displacement in the opposite direction of magnetic deflection. This effect is only weakly seen in the $\sim 7-\mathrm{MeV}$ image, but was qualitatively reproducible in $\sim 2-\mathrm{MeV}$ images from multiple shots. Lineouts from the four radiographs are shown in Figure $6 \mathrm{~b}$ where the solid lines are from the images of the 2 -T field. Some focusing may be expected due to the fall-off of the B-field with radius, but the net deflection would have been downward if this was the origin of the focusing. ${ }^{4}$

This unexpected result is still under investigation, but the general hypothesis is that a negative potential is generated by electrons trapped in the magnetic field. In this geometry, if $\gtrsim 10^{12}$ electrons are trapped, negative potentials of $\gtrsim 1 \mathrm{MV}$ are possible and at these levels would strongly affect trajectories of protons with energies less than a few $\mathrm{MeV}$. At a field strength of $2 \mathrm{~T}$, electrons with energies $\lesssim 300 \mathrm{keV}$ have gyro radii $\lesssim 1 \mathrm{~mm}$, and therefore could be trapped in the magnetic mirror created by the solenoid.

Using the analytic model of the solenoid B-field, trajectory calculations were performed for electrons of various energies and initial velocities. It was found that electron trajectories originating at the short-pulse irradiated target, $20 \mathrm{~mm}$ from the solenoid axis, were affected by the presence of the B-field. The trajectories of the highest energy electrons are not altered by the weak, solenoid fringe-fields near the target, but they do become deflected

\footnotetext{
${ }^{3}$ For reference, a $2-\mathrm{MeV}$ proton traversing a uniform 2-T B-field 5 - $\mathrm{mm}$ long would be deflected by $\sim 0.9 \mathrm{~mm}$ in the target plane of this geometry.

${ }^{4}$ Proton radiographs of mesh targets verified the B-field direction by demonstrating downward deflections at high proton energies. Though, low energy protons still showed strong focusing in the opposite direction.
}

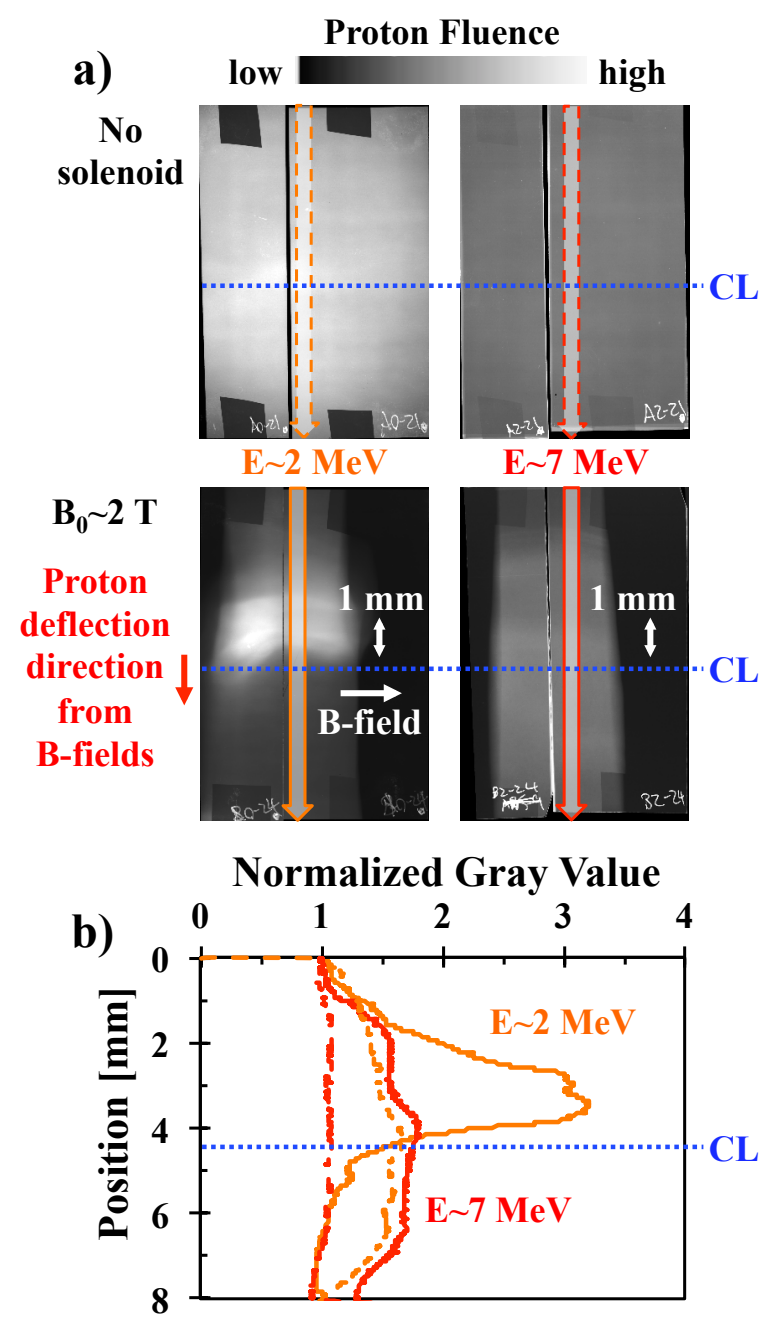

Figure 6: a) Proton radiographs with dominant energy sensitivities at $\sim 2$ and $\sim 7 \mathrm{MeV}$ for a case without the solenoid and for a case with a peak field of $2 \mathrm{~T}$. The centerline (CL) shows the vertical location of the backlighter. Tape shadows are visible, but of no consequence. b) Lineouts from radiographs with (solid) and without (dashed) an applied field. Focusing of protons is observed in the direction opposite to that expected from simple B-field deflections. Spatial scale is given in the target plane.

near the peak field in the gap between the coil. Due to the adiabatic nature of the trapping process, none of the short-pulse-generated electrons could be trapped near the peak field; magnetized electrons need to start near the axis to become properly trapped. A large supply of electrons near this location is available from the solenoid structure itself. It must be noted here that TNSA protons are accelerated within the first $100 \mu \mathrm{m}$ from the target surface by the electrostatic sheath field that is created by the highenergy-electrons leaving the target. Since the applied Bfield is weak enough at the backlighter target not to effect the trajectories of these electrons, the acceleration of the protons is not affected by the B-field and the protons become ballistic shortly after leaving the target.

Electrons emitted near the inner radius of the support structure at $\sim 3 \mathrm{~mm}$ may be readily trapped by the 
solenoid field. The short-pulse interaction with the backlighter target generates a large x-ray flash. These photons can penetrate the solenoid coils and support structure with a high enough flux to saturate image plates placed $\sim 30 \mathrm{~mm}$ away. Ablated plasma from x-ray energy deposition on the inner surface could yield a large enough population of magnetized electrons near the axis to be trapped in the mirrored field. This mechanism is still under investigation, but the fact that proton radiographs demonstrate the existence of a field structure other than the expected B-field is evident from data shown in Figure 6. This is an important observation for other researchers utilizing large solenoid structures to magnetize HED plasmas and something to consider when designing or activating an experimental platform.

\section{Plasma Jet Formation and Characterization}

\subsection{Numerical Modeling}

Radiation-hydrodynamic simulations using CRASH [25] demonstrate jet formation and collimation. CRASH is a hydrodynamic Eulerian code utilizing multi-level adaptive mesh refinement (AMR) with diffusive models for radiation transport and electron heat-transport. The experimental setup discussed in Section 2.1 was modeled in CRASH. The affect of laser energy on jet formation and morphology was numerically investigated. Figure 7 illustrates density distributions from these numerical experiments. Slight differences in jet morphology are apparent in Figure 7, though all simulations predict jet formation with a radius of $\sim 200 \mu \mathrm{m}$ at $50 \mathrm{~ns}$. For drive energies of $\geq 250 \mathrm{~J}$, simulations predict tip propagation up to $\sim 2.5 \mathrm{~mm}$, though the $150 \mathrm{~J}$ case generates slightly slower $(\sim 20 \%)$ jets with the jet tip propagating to $\sim 2 \mathrm{~mm}$. Due to the limitations of the optical probe beam, the densest regions of the jet will not be accessible for characterization. The optical diagnostics will effectively probe the lower density, higher temperature jet-tip.

\subsection{Interferometry Analysis}

An optical probe beam diagnosed the spatial structure of the collimated plasma flows, using the Mach-Zehnder interferometry configuration schematically shown in Figure 2 . The $\lambda_{p}=0.532-\mu \mathrm{m}$ probe was focused on to a PIMAX2 $512 \times 512$ CCD running with a $\sim 2$-ns temporal gate. The pixels on this camera have an effective size of 24.2$\mu \mathrm{m} \times 24.2-\mu \mathrm{m}$ and the optical configuration provided a magnification of 1.47 , creating an effective $8.43-\mathrm{mm} \times 8.43-\mathrm{mm}$ field-of-view at the target plane with $\sim 16.5$ - $\mu$ m spatial resolution. However, the spatial blurring due to the 2-ns gate is the dominant factor in determining the spatial resolution of the system, because the $\lesssim 50-\mu \mathrm{m} / \mathrm{ns}$ plasma moves $\lesssim 100 \mu \mathrm{m}$ during the temporal gate of the CCD. The absolute timing of the system was calibrated to the arrival time of the lasers to a total uncertainty of \pm 2 -ns, with contributions from all sources including the jitter in the

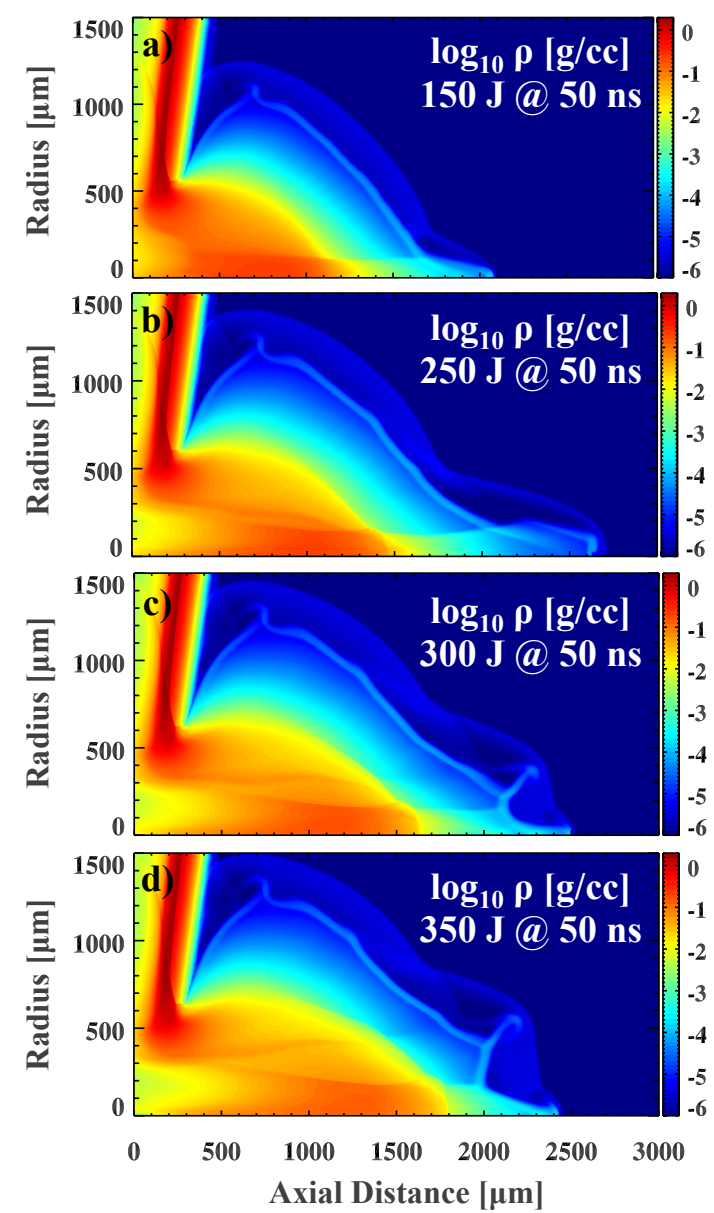

Figure 7: Density distributions at 50 ns as calculated by radiationhydrodynamic simulations using the CRASH code. Collimated jet formation is predicted from irradiated cone targets with a laser energy of a) $150 \mathrm{~J}, \mathrm{~b}) 250 \mathrm{~J}, \mathrm{c}) 300 \mathrm{~J}$, and d) $400 \mathrm{~J}$.

triggers and absolute beam times. In summary, the interferometry system fielded in these experiments provided interferograms with 2-ns temporal resolution, and a $\sim 70$ $\mathrm{mm}^{2}$ field-of-view with $\sim 0.1$-mm spatial resolution. ${ }^{5}$

Fringe shifts measured in the interferogram are created by a phase shift in the probe beam relative to the reference beam. This phase shift $(\Delta \phi)$ contains information about the plasma as it is directly related to the path integral of the index of refraction $(N)$ by

$$
\Delta \phi \approx k_{p} \int(N-1) d l,
$$

where $k_{p}$ is the vacuum wave number of the probe beam. The phase shift is retrieved from the interferograms through a wavelet analysis performed using the freely available Neutrino [26] software.

\footnotetext{
${ }^{5}$ The $\sim 125 \mu \mathrm{m}$ fringe wavelength (in the target plane) crossed more than 7 pixels of the CCD. Phase changes are easily measured down to two pixels, thus the $\sim 100 \mu \mathrm{m}$ spatial blurring is the dominant contributor to the resolution limit.
} 
a)

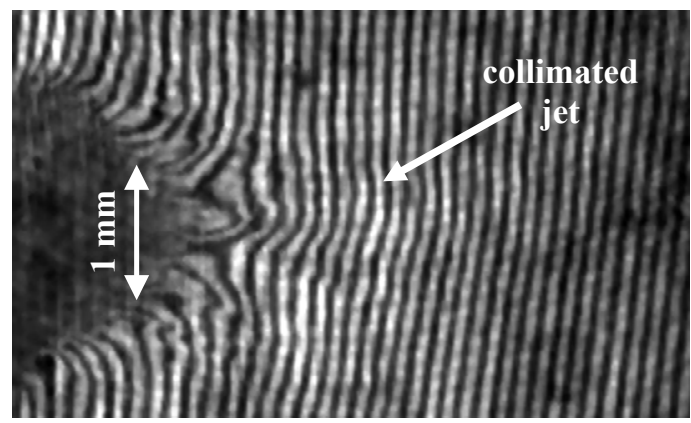

b)

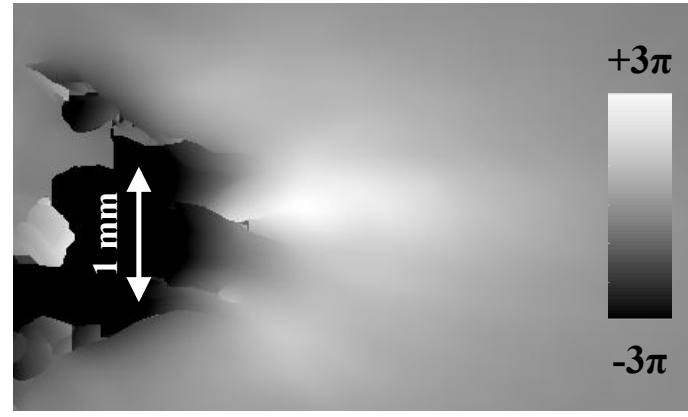

Figure 8: a) A sample jet interferogram. b) The retrieved phase image using the wavelet analysis in Neutrino. Phase information from the shadowed region is a numerical artifact and unimportant.

Figure 8 demonstrates a sample input and output of the wavelet analysis using Neutrino. Fringe shifts associated with the collimated jet are indicated in Figure 8a and the shadowed region shows where the probe beam is absorbed due to high path-integrated densities. It is clear that fringes in the plasma jet are shifted to the right, whereas near the shadow, fringes are bent to the left. This observed effect represents a change in sign of $\Delta \phi$. Figure $8 \mathrm{~b}$ shows the resulting $\Delta \phi$ map with phase changes ranging from $\pm 3 \pi$. Though phase information retrieved in the shadow is unimportant, fringes bent to the left near the shadow still contain relevant phase information.

The absolute sign of $\Delta \phi$ in a given image is inconsequential, however the relative change in sign of $\Delta \phi$ in a single image is important to note here. In general, the measured phase change is due to alterations in the index of refraction due to free electrons in the plasma as well as bound electrons in neutral, or partially ionized, gas. Moreover, the two effects act in opposite directions, specifically $N_{\text {gas }}>1$ and $N_{\text {plasma }}<1$ by the following equations,

$$
\begin{gathered}
N_{\text {gas }} \approx \sqrt{1+\frac{3}{N_{A}} \sum_{j} R_{j} n_{j}}, \\
N_{\text {plasma }} \approx \sqrt{1-\frac{n_{e}}{n_{c}}} .
\end{gathered}
$$

The Lorentz-Lorenz formulation for a multi-species dilute gas and the cold-plasma approximation have been used for Equations 9 and 10, respectively. In Equation $9, R_{j}$ and $n_{j}$ are the molar refractivity and number density of the $j^{t h}$ species (including all possible ionization states)

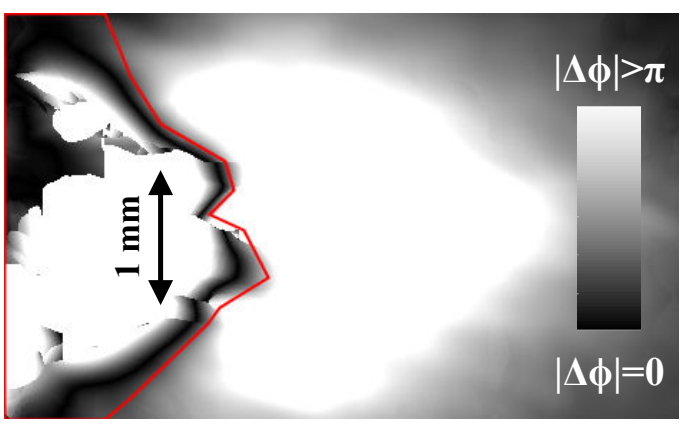

Figure 9: The absolute value of the $\Delta \phi$ distribution in Figure $8 \mathrm{~b}$ is shown. A well-defined $\Delta \phi=0$ surface is apparent.

and $N_{A}$ is Avogadro's number. In Equation $10, n_{e}$ is the free electron density and $n_{c}$ is the critical density determined by the vacuum wavelength $\left(\lambda_{\mu}\right.$ in microns) of the probe by $n_{c}=1.1 \times 10^{21} / \lambda_{\mu}^{2} \mathrm{~cm}^{-3}$. The index of refraction of partially ionized gases has been addressed by other researchers [27, 28] in terms of an 'average-atom' model for high-Z materials, and the opposing effects of free and bound electrons in low density $\left(n_{e} \sim 10^{14}-10^{15} \mathrm{~cm}^{-3}\right)$ plasmas were discussed recently by Merritt et al. [29].

Our data indicate a well-defined $\Delta \phi=0$ surface as shown in Figure 9. The measured phase change may be attributed to the sum of contributions from both bound and free electrons. The second term in each of Equations 9 and 10 are $<<1$, such that substituting into Equation 8 and summing the individual contributions, the total phase change may be approximated by

$$
\Delta \phi_{t o t} \approx k_{p} \int\left(\frac{3}{2 N_{A}} \sum_{j} R_{j} n_{j}-\frac{n_{e}}{2 n_{c}}\right) d l .
$$

Acquiring complete knowledge of the first term in Equation 11 is a monumental task, requiring molar refractivities at the probe wavelength and the number densities of all ionization states present in the plasma. A measurable contribution from bound electrons may be expected at low ionization states due to the low temperatures, of order $\lesssim 1 \mathrm{eV}$, as predicted by CRASH. For the purposes of this paper, bound-electron contributions to the index of refraction are neglected and regions near the $\Delta \phi=0$ surface are masked-out in the analysis; as represented by the outlined region in Figure 9 for that specific image. However, these results have prompted interest in further research to combine an 'average-atom'-like equilibrium model to constrain plasma conditions at the $\Delta \phi=0$ surface.

\subsection{Interferometry Results of Collimated Plasma Jets}

In many plasma experiments the index of refraction is completely dominated by the the free-electron contribution. This approach is taken in the following discussion on interferograms of collimated plasma jets with the caveat that the measured phase represents a lower estimate on the actual free-electron density since any bound contribution reduces the measured $\Delta \phi$ and, therefore the inferred 


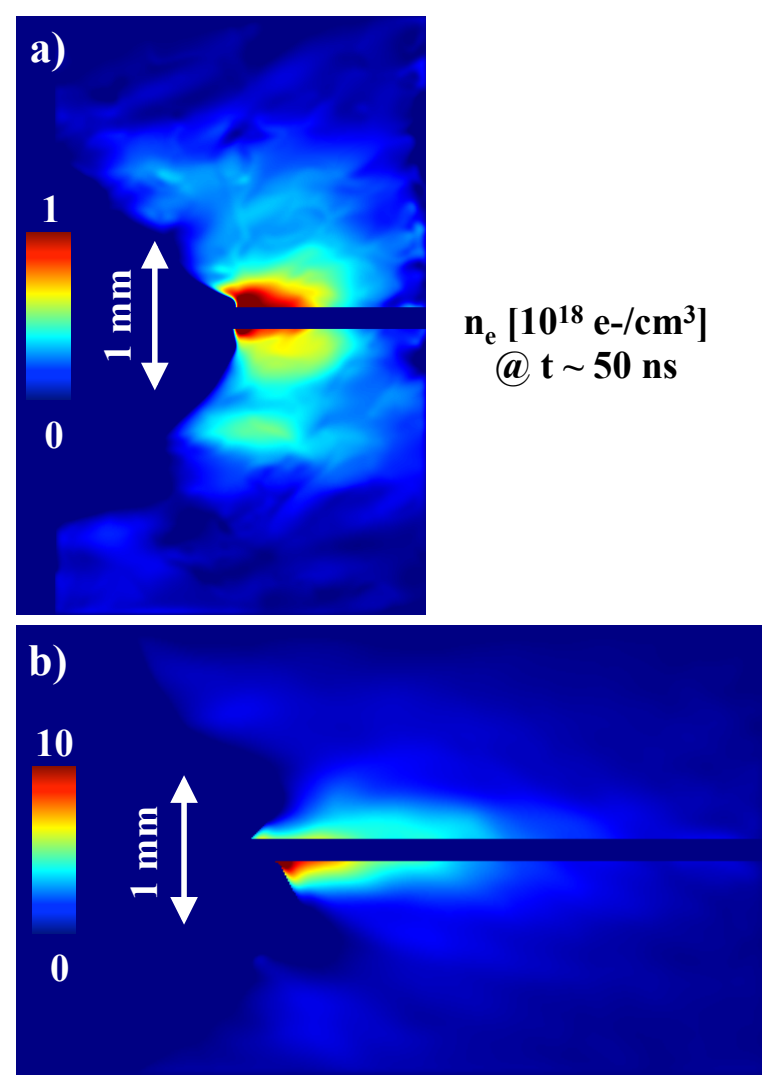

Figure 10: Electron density distributions (in $10^{18} \mathrm{~cm}^{-3}$ ) inferred from interferometry data taken at $\sim 50 \mathrm{~ns}$ after the onset of the 10-ns drive pulse with a) $\sim 180 \mathrm{~J}$ and b) $\sim 330 \mathrm{~J}$. Jet collimation is slightly better at higher drive intensity resulting in higher peak densities. An average jet velocity is estimated at $\sim 45 \mu \mathrm{m} / \mathrm{ns}$.

electron density. The free contribution to the phase change $\left(\Delta \phi_{e}\right)$ may be written as

$$
\Delta \phi_{e} \approx-\frac{k_{p}}{2 n_{c}} \int n_{e} d l
$$

To determine the electron densities, cylindrical symmetry is assumed and an Abel transform [30] performed on the $\Delta \phi_{e}$ distribution using the Neutrino software. Due to the nature of the inversion, the deconvolution noise approaches $100 \%$ at the axis of symmetry; radii $\lesssim 75 \mu$ m are masked to ensure that numerical error associated with the inversion is minimized in the inferred electron density distributions. The inversion is separately applied to both halves of the image and differences between the two halves are likely associated with azimuthal variation in the plasma. A typical error in the inferred electron density was estimated to be $\sim 30 \%$ by directly comparing the two halves in each image.

Irradiated plastic cones generated well-collimated, mmscale plasma flows as indicated by measured electron density distributions shown in Figure 10. The image in Figure $10 \mathrm{~b}$ is the same data discussed in Figures 8 and 9 and illustrates the masking associated with the shadow, with regions of substantial bound-electron contribution to $\Delta \phi$, and with high numerical noise at small radii. The drive laser energy was varied to investigate the effect of intensity on jet morphology. The data show that reducing the intensity from $\sim 1.2 \times 10^{13} \mathrm{~W} / \mathrm{cm}^{2}$ to $\sim 6.4 \times 10^{12} \mathrm{~W} / \mathrm{cm}^{2}$ slightly degrades the collimation and the peak electron density of the plasma jet. In both cases however, the densest area of the jet is collimated to a diameter of $\sim 500 \mu \mathrm{m}$, though the higher intensity drive produces collimation over a longer distance. Higher intensity drive also increases the inferred peak electron density by a factor of $\gtrsim 5$. This may be attributed to a combination of effects, such as target variation, higher ionization states associated with higher temperatures, and varying degrees of on-axis collimation.

Plasma pressure of observed collimated jets is estimated from measured and simulated values. For electron densities of $\sim 5 \times 10^{18} \mathrm{~cm}^{-3}$ and temperatures of order $\sim 1 \mathrm{eV}$, the thermal pressure is near $\sim 10$ bar. The velocity of the jet may be estimated using the distance traveled and the transit time of the flow. The densest regions of the plasma are $\sim 2.2 \mathrm{~mm}$ from the cone apex ${ }^{6}$ after traveling for $\sim 50 \mathrm{~ns},{ }^{7}$ resulting in an average jet velocity of $\sim 45 \mu \mathrm{m} / \mathrm{ns}$. At an ionization state of order unity, the mass density of the jet is estimated to be $\sim 50 \mu \mathrm{g} / \mathrm{cm}^{3}$ resulting in a ram pressure of $\rho v^{2} \sim 10^{3}$ bar. The plasma kinetic energy is dominated by the ram pressure, as is often the case for astrophysical jets. It is important to note again, that these are lower estimates of the density due to the neglected bound-electron contribution in the interferograms as discussed earlier. However, these calculations provide a good estimate of actual jet parameters achieved with these targets and laser conditions.

\subsection{Proton Radiography of Collimated Plasma Jets}

Short-pulse proton radiography probed plasma flows for self-generated fields. Noncollinear density and temperature gradients in the plasma create B-fields through the so-called Biermann battery source and gradients in electron pressure generate electric fields, [31]

$$
\begin{gathered}
\frac{\partial \mathbf{B}}{\partial t} \approx \frac{\nabla T_{e} \times \nabla n_{e}}{e_{0} n_{e}}, \\
\mathbf{E} \approx-\frac{\nabla p_{e}}{e_{0} n_{e}},
\end{gathered}
$$

where $T_{e}$ and $n_{e}$ are the electron temperature and density distributions respectively, and $e_{0}$ is the unit charge. Protons are sensitive to fields in the plasma through deflections caused by the Lorentz force as discussed in Section 3.3 for the case of the solenoid field.

Proton radiographs of these jets show no significant self-generated fields near the collimated plasma flow measured from interferograms. However, proton images do

\footnotetext{
${ }^{6}$ This distance estimate is from the back (non-irradiated) side of the cone and includes the $\sim 440 \mu \mathrm{m}$ distance out of the interferometry field of view.

${ }^{7}$ The predicted shock transit time through the $90 \mu \mathrm{m}$-thick cone is $\sim 2$-ns and is ignored for estimation purposes at times near 50 ns.
} 

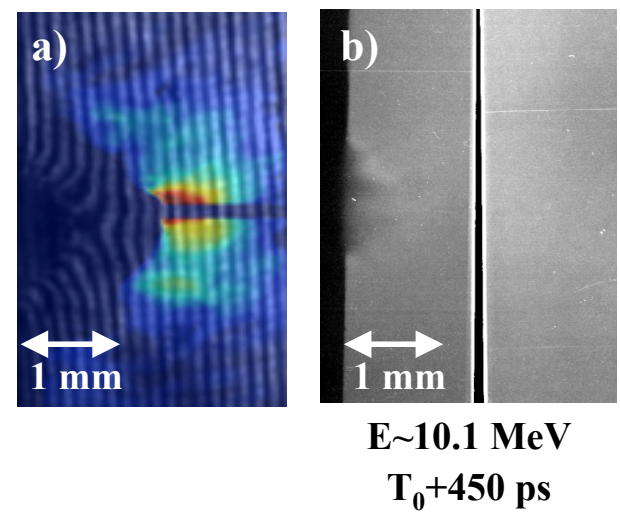

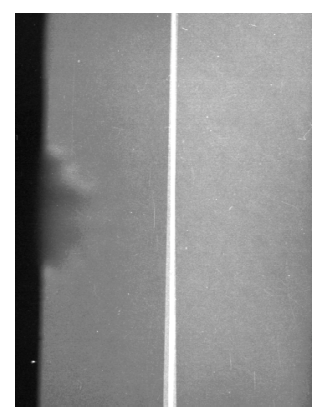

$\mathrm{E} \sim 7.6 \mathrm{MeV}$
$\mathrm{T}_{\mathbf{0}}+520 \mathrm{ps}$

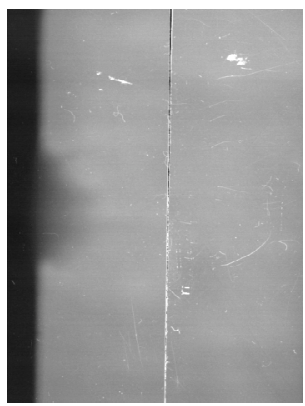

E 5.0 MeV

$\mathrm{T}_{0}+650 \mathrm{ps}$

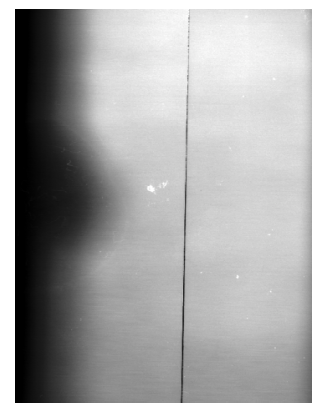

E 2.3 MeV $\mathrm{T}_{0}+950 \mathrm{ps}$
Proton

Fluence

high

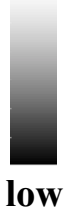

Figure 11: a) Interferogram overlaid on the electron density distribution shown in Figure 10a to illustrate the location of the collimated jet. b) Proton images at various energies taken in a direction orthogonal to the interferometry axis. Structure is only observed in the cold, dense regions corresponding to the shadow in inteferometry data. The time of flight difference for each proton image is shown where $\mathrm{T}_{0}$ is $\sim 50$ ns. The break in each image is due to the slit for the proton spectrometer.

reveal structure in the cold, dense region of the flow. Figure 11a shows the electron density distribution from Figure 10a overlaid on the raw interferogram to illustrate the location of the observed jet; $\sim 1.8 \mathrm{~mm}$ from the target edge (not the cone apex). Proton radiographs from this same shot are shown in Figure 11b on the same spatial scale with structure appearing only within $\sim 1 \mathrm{~mm}$ from the target surface, as labeled.

Trajectory deflections are inversely related to proton energy when caused by electric $\left(\theta_{E} \propto 1 / E_{p}\right)$ and magnetic $\left(\theta_{B} \propto 1 / \sqrt{E_{p}}\right)$ fields [31]. Lower energy protons are more susceptible to deflections from fields as well as Coulomb scattering than higher energy protons; as evident by the diffusive target edge and lack of structure in the $\sim 2.3 \mathrm{MeV}$ image. At higher energies, proton images show faint striation-like structure with some observed proton enhancement that may be caused by self-generated fields or Coulomb scattering. These mm-size features, if caused by fields, would indicate path-integrated field strengths of order $\sim 1$ T-mm or $\sim 10^{4}-10^{5} \mathrm{~V}$ for $\mathrm{B}$ and $\mathrm{E}$ fields, respectively. Future analysis will take into consideration the exact proton spectra from the backlighter target.

The observed structure is likely related to the expanding cold, plastic cone, and therefore unimportant to the collimated plasmas flows of interest in this work. These data suggest that the observed collimated flows are due to the hydrodynamics of the system and that self-generated fields do not play a role in their evolution.

\section{Magnetized Plasma Jets}

Collimated plasma flows were magnetized using the solenoid field discussed in Section 3 and characterized with interferometry. ${ }^{8}$ The applied magnetic field was aligned in

\footnotetext{
${ }^{8}$ Proton radiography of magnetized plasma jets was attempted, but complications discussed in Section 3.3 prohibit accurate interpretation of the data at this time.
}

the same direction as the bulk motion of the flow with a peak field strength of 5 Tesla. The magnetic energy density associated with this field strength is $\sim 10^{2}$ bar. Since the plasma energy is dominated by the ram pressure $\left(\sim 10^{3}\right.$ bar $)$, the ratio of plasma energy to magnetic energy $(\beta)$ was $\sim 10$; magnetic pressures of $\sim 10^{3}$ require a $\sim 16 \mathrm{~T}$ field. However, other figures of merit should also be considered in assessing potential effects associated with B-fields.

\subsection{The Hall Parameter}

The Hall parameter $(\chi)$ characterizes the level of magnetization in a plasma. It is a quantity that describes the characteristic number of field gyrations before a Coulombic collision. A Hall parameter of $\sim 1$ indicates moderate magnetization as trajectories are completely altered by the field before randomization by collisions. As electrons are easier to magnetize due to their low mass, $\chi$ is typically calculated as the product of the electron cyclotron frequency $\left(\omega_{c e}\right)$ and the electron-ion collision time $\left(\tau_{e i}\right)$,

$$
\chi_{e}=\omega_{c e} \tau_{e i} .
$$

Using a typical definition for the collision time in a thermalized plasma, this can be approximated by

$$
\chi_{e} \approx\left(\frac{e_{0} B}{m_{e}}\right)\left(\frac{12 \pi^{3 / 2} \epsilon_{0}^{2}}{\sqrt{2} e_{0}^{4}} \frac{\sqrt{m_{e}} T_{e}^{3 / 2}}{n_{e} Z \ln \Lambda}\right) .
$$

This expression invokes the use of the Coulomb logarithm $(\ln \Lambda)$ which, in these cold plasmas, is difficult to accurately define. However, it is relatively insensitive to plasma parameters and, for estimation purposes discussed here, it is assumed to be 2 . This results in the following simple expression in practical units,

$$
\chi_{e} \approx 0.03 \frac{B_{T} T_{e, e V}^{3 / 2}}{n_{e, 18} Z}
$$




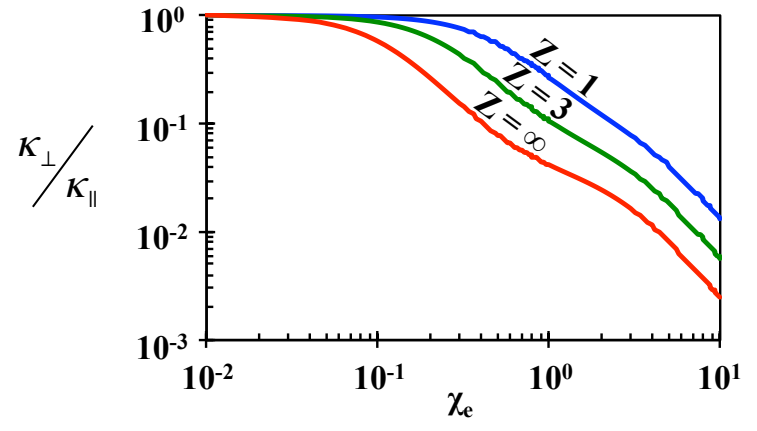

Figure 12: Electron heat conduction perpendicular to the field is reduced relative to parallel heat conduction as described by Equation 18. The three lines were generated using the Braginskii coefficients [32] for the labeled $\mathrm{Z}$ values.

where the magnetic field is in Tesla, the electron temperature is in $\mathrm{eV}$, the electron density is in $10^{18} \mathrm{~cm}^{-3}$, and $Z$ is the characteristic ionization state. A $5 \mathrm{~T}$ magnetic field generates a Hall parameter of $\sim 0.15$ when all other parameters in Equation 17 are of order unity; indicating a weakly magnetized electron population. ${ }^{9}$ Under these conditions, it may be expected that electron trajectories will be affected by the magnetic field, but that collisions with background ions will be frequent.

Heat conduction by plasma electrons may still be affected by the presence of the magnetic field, even in the weakly magnetized $\left(\chi_{e}\right)$ regime. The description of collisional heat transport across magnetic field lines has been described by Braginskii [32] who gives the ratio of perpendicular to parallel heat conduction $\left(\kappa_{\perp} / \kappa_{\|}\right)$as

$$
\frac{\kappa_{\perp}}{\kappa_{\|}}=\frac{\gamma_{1}^{\prime} \chi_{e}^{2}+\gamma_{0}^{\prime}}{\gamma_{0}\left(\chi_{e}^{4}+\delta_{1} \chi_{e}^{2}+\delta_{0}\right)},
$$

where the coefficients $\gamma_{1}^{\prime}, \gamma_{0}^{\prime}, \gamma_{0}, \delta_{1}, \delta_{0}$ are given by Braginskii as a function of Z . Figure 12 illustrates the strong variation in heat conduction for $\chi_{e}$ up to 10 . For the estimated conditions already discussed and an ionization state near unity, the expected heat conduction across field lines is still $\sim 90 \%$ of the parallel value; a weak effect given assumptions in the calculation. However, the spatial variation in the electron density alters the local Hall parameter, and thus the lower density (higher temperature) regions may confine the perpendicular heat flux more efficiently.

\subsection{Experimental Results}

Complete disruption of hydrodynamically collimated jets occurred when the external magnetic field was applied. Instead, a hollow cavity structure was observed. Figure 13 illustrates the difference in jet morphology at $\sim 50 \mathrm{~ns}$ as measured from interferometry. For a simple comparison Figure 10a, the unmagnetized electron density distribution, is reproduced next to that of the magnetized flow in

\footnotetext{
${ }^{9}$ Under these conditions, the cyclotron frequency and collision frequency are $\sim 0.9 \mathrm{ps}^{-1}$ and $6 \mathrm{ps}^{-1}$, respectively.
}
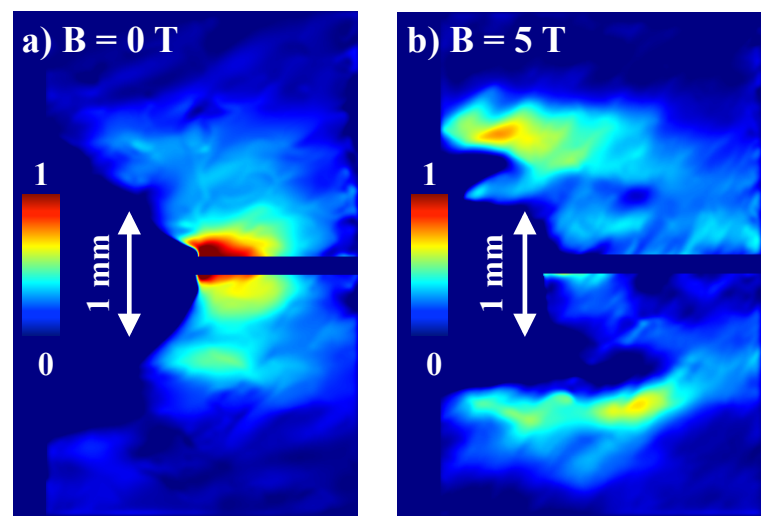

Figure 13: Electron density distributions obtained from interferometry data at $\mathrm{t} \sim 50 \mathrm{~ns}$ for the case of a) no B-field and b) an applied B-field of $5 \mathrm{~T}$. The magnetic field completely disrupts the collimated jet to form a hollow cavity.

Figure 13. The flow is still collimated when magnetized, though rather than a solid jet with a $\sim 500 \mu$ m diameter, the plasma takes on the shape of a hollow cylindrical cavity that tapers from a diameter of $\sim 2.9 \mathrm{~mm}$ down to $\sim 1.9 \mathrm{~mm}$ over a $\sim 2$-mm distance. The thickness of the cavity wall is $\sim 300 \mu \mathrm{m}$, slightly thinner than the unmagnetized flow.

Electron particle diffusion across field lines may be characterized by the Larmor motion. The gyro radius of a $1 \mathrm{eV}$ electron in a $5 \mathrm{~T}$ field is $r_{e} \sim 0.34 \mu \mathrm{m}$ which is much smaller than the unmagnetized, collimated jet $(\sim 500 \mu \mathrm{m})$. This suggests that many collisions, and therefore many fractions of field gyrations due to the low Hall parameter, are necessary to act across the spatial scales of interest; 'random walk' motion. The length scale associated with cross-field diffusion may be estimated by,

$$
L_{e} \approx \sqrt{D_{e} t}
$$

where $D_{e}=r_{e}^{2} / \tau_{e i}$ is the diffusion coefficient with timescale $t$. For the estimates discussed here, the approximate diffusion scale length is of order $\sim 200 \mu \mathrm{m}$. This scale is consistent with the observed envelope thickness suggesting a diffusive process, however, this is much smaller than the few-millimeter scale of the envelope diameter.

Magnetized jet morphology is different from the unmagnetized case. The unmagnetized ram pressure in the axial direction of the plasma would be $\sim 10$ times that of the 5 - $\mathrm{T}$ B-field $(\beta \sim 10)$. In the magnetized case, however, the mass accumulates radially with a ram pressure that is $\sim 4$ times less than that in the axial, unmagnetized case. The reduced ram pressure in the radial direction, along with some potential compression of the field in the envelope, creates local plasma conditions near the $\beta \sim 1$ regime; where the magnetic field may strongly affect the dynamics.

\subsection{Physics Discussion}

Numerical work by Ciardi et al. [5] predicts the formation of a cavity surrounded by a shock envelope in magnetically collimated jets. In their simulations, a faster, hotter 
flow expands in a strong axial magnetic field. The envelope is generated through radial deceleration (pinching effect) of the expanding plasma due to the Lorentz force, $F_{r}=j_{\theta} B_{z}$, on the induced current $j_{\theta}$. Magnetic fields are swept up by the flow due to low resistivity in the hot plasma. A conical shock forms, leading to an accumulation of plasma on-axis that produces an axial jet $\sim 15 \mathrm{~mm}$ away from the initial target surface; much farther than was probed in our experiments. Further propagation of the jet results in a series of compressions and expansions that produce shock structures. It is also shown that increasing the field strength for fixed initial plasma conditions results in tighter jet formation closer to the target surface.

There are qualitative similarities between experimental results discussed here and the plasmas numerically investigated by Ciardi et al., despite significant differences in plasma parameters. Figure 13b shows the envelope structure caused by the applied magnetic field. With the applied field, the fast region of the flow is still near magnetosonic as the magnetoacoustic velocity $\left(c_{m a}=\sqrt{c_{A}^{2}+c_{s}^{2}}\right)$ is $\sim 20 \mu \mathrm{m} / \mathrm{ns}$, and weak shocks may form. Observed tapering of the cavity may be due to radial deceleration of expanding plasma and jet formation may have been observed further downstream as predicted by Ciardi et al., however the field-of-view did not permit verification. This description of the morphology is consistent with the observations, though differences in plasma conditions must be noted.

Unlike the naturally expanding plasma discussed by Ciardi et al., the cone geometry used in these experiments directs plasma inwards to form the collimated jet. Some plasma does expand outwards and could be forced inward by the applied field to form the shock envelope, but the background B-field also prevents the formation of the original collimated outflow. Moreover, under these plasma conditions, the magnetic Reynolds number $\left(R e_{m}\right)$ is of order $\sim 1$ with a typical diffusion time $\left(\tau_{\text {diff }}\right)$ of order $\sim 0.1 \mathrm{~ns}$, indicating non-negligible magnetic diffusion effects over dynamical timescales.

If the shock envelope is formed due to radial deceleration, B-fields will not pile up at the shock location. Rather, fields will diffuse through the plasma on timescales much shorter than those of the dynamics of the system, though they still dramatically affect the plasma evolution. Due to the low $R e_{m}$, these plasmas are not accurately described by the Euler MHD equations, which makes their dynamic evolution different than that of the analog astrophysical system. Using thinner targets and higher laser energy will likely help to make this platform more closely analogous to astrophysical jets by increasing the flow speed to increase the mach number of the flow and raising the plasma temperature to reduce diffusive effects.

Qualitatively similar behavior is observed in our experiments as in the system numerically investigated by Ciardi et al. that is described well by the Euler MHD equations. The deceleration mechanism that forms the cavity-shock structure is caused by the induced current in the expand- ing plasma. The radially converging areas of the plasma in our experiments, in a similar manner, may induce a current in the opposite direction thereby producing an outward (rather than inward) Lorentz force. This current configuration would allow the formation of the outer shock envelope while simultaneously preventing the inner flow to properly collimate. Future work with these data will focus on detailed modeling of the system including B-field effects and resistivity to investigate cavity formation and collimated jet disruption.

\section{Summary}

Magnetized-jet experiments were successfully executed and demonstrated dramatic effects on collimated outflows in the moderate $\beta$ regime. Irradiated plastic cones were shown to produce hydrodynamically collimated jets with a diameter of $\sim 500 \mu \mathrm{m}$ that propagate up to $\sim 2.5 \mathrm{~mm}$. Variation in laser energy by a factor of $\sim 1.8$ produced minimal differences in jet collimation, with the expected result that flows were slightly slower at lower energies. Proton radiography of these outflows suggested that self-generated fields did not play a significant role in jet collimation. A customdesigned solenoid provided a uniform 5-T magnetic field aligned with the outflow to magnetize the plasma. Spatial variation in the B-field was characterized using a multiloop b-dot probe and verified a $\lesssim 5 \%$ variation in field strength across the region of plasma flow.

Complete disruption of the collimated jet was observed when the $5 \mathrm{~T}$ magnetic field was applied. A hollow cavity developed in its place, as was predicted for hotter, faster outflows in previous numerical work by Ciardi et al. We propose that the diamagnetic forces that create the envelope, switch directions at the converging regions of our plasma thereby preventing the collimation of the jet. One important difference between our experiments and the previous simulations is that our resistivity is much higher due to the lower plasma temperatures. This likely plays a role in the evolution of the magnetic field and diamagnetic current profile within the jet and is the focus of future numerical work based on these experiments.

\section{Future Work}

A number of unanticipated results from this work have stimulated interest for further investigation. These have been mentioned in the main body of the text, but are reiterated here for clarity. The most pressing interest is the further development of the CRASH code for simulating magnetic field effects. Specifically to help explain jet disruption due to background magnetic fields and the role plasma resistivity may play in this effect.

In this work we radiographed a known B-field structure with unexpected results as discussed in Section 3.3. Proton radiography has been used to infer magnetic fields in many different experiments and a good understanding of 
these data may help future use of this diagnostic technique. Analysis of spectral data from the imaging spectrometer also fielded in these experiments may lead to valuable trajectory information that can help to explain the unanticipated focusing effect that was observed.

Interferometry data taken of these jets showed a measurable contribution to the phase shift from bound electrons. A complete description of the index of refraction including all possible ionization states is a futile task with the diagnostic suite fielded for these experiments. However, the surface at which point the phase change is zero may provide a path to inferring local plasma conditions using analytic models and equilibration assumptions. This technique may be useful in further constraining numerical models and provide another measure of plasma conditions.

This experimental platform has produced good results and may be used for future magnetized jet experiments. To connect better with astrophysical systems, the next generation targets should be thinner such that the flow velocity and plasma temperature may increase enough to make magnetic diffusion negligible. Also, the magnetic field orientation relative to the outflow should be varied to investigate the effects of field alignment. The simplest experiment would be to flip the polarity of the solenoid such that the field would be antiparallel to the flow. With this solenoid configuration, it is also straightforward to align the field perpendicular to the flow. Interactions between colliding jets at different angles within a magnetic field is also a potential direction of future research.

\section{Acknowledgements}

The authors express their gratitude to the staff at the Jupiter Laser Facility for a successful experimental campaign. M. J.-E. Manuel also thanks Tommaso Vinci and Alessandro Flacco at LULI for the use of the Neutrino software and useful conversations regarding interferometry analysis and Abel inversion techniques. Special thanks is given to Robb Gillespie for support in construction techniques used for the solenoid coils and housing.

Other support for this work was provided by NASA through Einstein Postdoctoral Fellowship grant number PF3-140111 awarded by the Chandra X-ray Center, which is operated by the Astrophysical Observatory for NASA under contract NAS8-03060, by the NNSA-DS and SCOFES Joint Program in High-Energy-Density Laboratory Plasmas, grant number DE-NA0001840 and by the Predictive Sciences Academic Alliances Program in NNSA-ASC via grant DEFC52-08NA28616. Work by LLNL was performed under the auspices of U.S. DOE under contract DE-AC52-07NA27344.

[1] R. L. Curran and A. Chrysostomou. "Magnetic fields in massive star-forming regions". Monthly Notices of the Royal Astronomical Society, 382, 699 (2007). doi:10.1111/j.13652966.2007.12399.x.

[2] J. Ferreira and R. Deguiran. "What does it take to launch self-confined jets from astrophysical accretion discs?" High Energy Density Physics, 9(1), 67 (2013). doi:10.1016/j.hedp.2012.09.012.

[3] C. Carrasco-González, L. F. Rodríguez, G. Anglada, J. Martí, J. M. Torrelles, and M. Osorio. "A Magnetized Jet from a Massive Protostar". Science, 330, 1209 (2010). doi:10.1126/science.1195589.

[4] A. P. Marscher, S. G. Jorstad, F. D. D'Arcangelo, P. S. Smith, G. G. Williams, V. M. Larionov, H. Oh, A. R. Olmstead, M. F. Aller, H. D. Aller, I. M. McHardy, A. Lähteenmäki, M. Tornikoski, E. Valtaoja, V. A. Hagen-Thorn, E. N. Kopatskaya, W. K. Gear, G. Tosti, O. Kurtanidze, M. Nikolashvili, L. Sigua, H. R. Miller, and W. T. Ryle. "The inner jet of an active galactic nucleus as revealed by a radio-to- $\gamma$-ray outburst". Nature, 452, 966 (2008). doi:10.1038/nature06895.

[5] A. Ciardi, T. Vinci, J. Fuchs, B. Albertazzi, C. Riconda, H. Pépin, and O. Portugall. "Astrophysics of Magnetically Collimated Jets Generated from Laser-Produced Plasmas". Physical Review Letters, 110(2), 025002 (2013). doi:10.1103/PhysRevLett.110.025002.

[6] D. D. Ryutov, R. P. Drake, J. Kane, E. Liang, B. A. Remington, and W. M. Wood-Vasey. "Similarity Criteria for the Laboratory Simulation of Supernova Hydrodynamics". The Astrophysical Journal, 518, 821 (1999). doi:10.1086/307293.

[7] P. Nicolaï, C. Stenz, V. Tikhonchuk, A. Kasperczuk, T. Pisarczyk, L. Juha, E. Krousky, K. Masek, M. Pfeifer, K. Rohlena, J. Skala, V. Kmetik, J. Ullschmied, M. Kalal, D. Klir, J. Kravarik, P. Kubes, K. Rezac, P. Pisarczyk, and E. Tabakhoff. "Experimental evidence of multimaterial jet formation with lasers". Physics of Plasmas, 17(11), 112903 (2010). doi:10.1063/1.3511774.

[8] S. P. Nikitin, J. Grun, Y. Aglitskiy, C. Manka, D. Zabetakis, A. L. Velikovich, and C. Miller. "Production of cumulative jets by ablatively-driven implosion of hollow cones and wedges". Physics of Plasmas, 15(5), 050703 (2008). doi:10.1063/1.2917917.

[9] C. D. Gregory, A. Diziére, H. Aoki, H. Tanji, T. Ide, E. Falize, B. Loupias, C. Michaut, T. Morita, S. A. Pikuz, A. Ravasio, Y. Kuramitsu, Y. Sakawa, H. Takabe, N. C. Woolsey, and M. Koenig. "Experiments to investigate the effects of radiative cooling on plasma jet collimation". High Energy Density Physics, 11(0), 12 (2014). doi:10.1016/j.hedp.2013.12.003.

[10] W. S. Rasband. "ImageJ". U.S. National Institutes of Health (1997-2012).

[11] C. A. Schneider, W. S. Rasband, and K. W. Eliceiri. "NIH Image to ImageJ: 25 years of image analysis". Nature Methods, $\mathbf{9}(7), 671$ (2012). doi:10.1038/nmeth.2089.

[12] S. Klein. Fusion Science and Technology, to be submitted (2014).

[13] A. J. Mackinnon, P. K. Patel, R. P. Town, M. J. Edwards, T. Phillips, S. C. Lerner, D. W. Price, D. Hicks, M. H. Key, S. Hatchett, S. C. Wilks, M. Borghesi, L. Romagnani, S. Kar, T. Toncian, G. Pretzler, O. Willi, M. Koenig, E. Martinolli, S. Lepape, A. Benuzzi-Mounaix, P. Audebert, J. C. Gauthier, J. King, R. Snavely, R. R. Freeman, and T. Boehlly. "Proton radiography as an electromagnetic field and density perturbation diagnostic (invited)". Review of Scientific Instruments, 75(10), 3531 (2004). doi:10.1063/1.1788893.

[14] A. U. Hazi. Applied Physics Letters, to be submitted (2014).

[15] H. Chen, A. U. Hazi, R. van Maren, S. N. Chen, J. Fuchs, M. Gauthier, S. Le Pape, J. R. Rygg, and R. Shepherd. "An imaging proton spectrometer for short-pulse laser plasma experiments". Review of Scientific Instruments, 81(10), 10D314 (2010). doi:10.1063/1.3483212.

[16] A. Rasmus. Review of Scientific Instruments, to be submitted (2014).

[17] S. Klein. Review of Scientific Instruments, to be submitted (2014).

[18] E. E. Callaghan and S. H. Maslen. "The Magnetic Field of a Finite Solenoid". Technical report, NASA (1960).

[19] F. Bitter. "The Design of Powerful Electromagnets Part IV. The New Magnet Laboratory at M. I. T". Review of Scientific 
Instruments, 10(12), 373 (1939). doi:10.1063/1.1751470.

[20] B. B. Pollock, D. H. Froula, P. F. Davis, J. S. Ross, S. Fulkerson, J. Bower, J. Satariano, D. Price, K. Krushelnick, and S. H. Glenzer. "High magnetic field generation for laser-plasma experiments". Review of Scientific Instruments, 77(11), 114703 (2006). doi:10.1063/1.2356854.

[21] A. B. Zylstra, C. K. Li, H. G. Rinderknecht, F. H. Séguin, R. D. Petrasso, C. Stoeckl, D. D. Meyerhofer, P. Nilson, T. C. Sangster, and S. Le Pape. "Using high-intensity laser-generated energetic protons to radiograph directly driven implosions". Review of Scientific Instruments, 83(1), 013511 (2012). ISSN 00346748. doi:10.1063/1.3680110.

[22] N. L. Kugland, J. S. Ross, P. Y. Chang, R. P. Drake, G. Fiksel, D. H. Froula, S. H. Glenzer, G. Gregori, M. Grosskopf, C. Huntington, M. Koenig, Y. Kuramitsu, C. Kuranz, M. C. Levy, E. Liang, D. Martinez, J. Meinecke, F. Miniati, T. Morita, A. Pelka, C. Plechaty, R. Presura, A. Ravasio, B. A. Remington, B. Reville, D. D. Ryutov, Y. Sakawa, A. Spitkovsky, H. Takabe, and H. S. Park. "Visualizing electromagnetic fields in laserproduced counter-streaming plasma experiments for collisionless shock laboratory astrophysics". Physics of Plasmas, 20(5), 056313 (2013). doi:10.1063/1.4804548.

[23] M. J.-E. Manuel, A. B. Zylstra, H. G. Rinderknecht, D. T. Casey, M. J. Rosenberg, N. Sinenian, C. K. Li, J. A. Frenje, F. H. Séguin, and R. D. Petrasso. "Source characterization and modeling development for monoenergetic-proton radiography experiments at OMEGA". Review of Scientific Instruments, 83(6), 063506 (2012). doi:10.1063/1.4730336.

[24] M. J.-E. Manuel, N. Sinenian, F. H. Séguin, C. K. Li, J. A. Frenje, H. G. Rinderknecht, D. T. Casey, A. B. Zylstra, R. D. Petrasso, and F. N. Beg. "Mapping return currents in laser-generated Z-pinch plasmas using proton deflectometry". Applied Physics Letters, 100(20), 203505 (2012). doi:10.1063/1.4718425.

[25] B. Van der Holst, G. Tóth, I. V. Sokolov, K. G. Powell, J. P.
Holloway, E. S. Myra, Q. Stout, M. L. Adams, J. E. Morel, and S. Karni. "CRASH: A Block-adaptive-mesh Code for Radiative Shock Hydrodynamics - Implementation and Verification". The Astrophysical Journal Supplement Series, 194(2), 23 (2011). ISSN 0067-0049. doi:10.1088/0067-0049/194/2/23.

[26] A. Flacco and T. Vinci. Neutrino v941t. Laboratoire d'Utilisation des Lasers Intenses (2013). Current version can be downloaded here.

[27] J. Nilsen, W. R. Johnson, C. A. Iglesias, and J. H. Scofield. "Impact of anomalous dispersion on the interferometer measurements of plasmas". Journal of Quantitative Spectroscopy and Radiative Transfer, 99(1-3), 425 (2006). doi:10.1016/j.jqsrt.2005.05.034.

[28] W. R. Johnson, C. Guet, and G. F. Bertsch. "Optical properties of plasmas based on an average-atom model". Journal of Quantitative Spectroscopy and Radiative Transfer, 99(1-3), 327 (2006). doi:10.1016/j.jqsrt.2005.05.026.

[29] E. C. Merritt, A. L. Moser, S. C. Hsu, C. S. Adams, J. P. Dunn, A. Miguel Holgado, and M. A. Gilmore. "Experimental evidence for collisional shock formation via two obliquely merging supersonic plasma jets". Physics of Plasmas, 21(5), 055703 (2014). doi:10.1063/1.4872323

[30] P. S. Kolhe and A. K. Agrawal. "Abel inversion of deflectometric data: comparison of accuracy and noise propagation of existing techniques". Applied Optics, 48(20), 3894 (2009). doi:10.1364/AO.48.003894

[31] M. J.-E. Manuel, C. K. Li, F. H. Séguin, J. A. Frenje, D. T. Casey, R. D. Petrasso, S. X. Hu, R. Betti, J. Hager, D. D. Meyerhofer, and V. Smalyuk. "Rayleigh-Taylor-induced magnetic fields in laser-irradiated plastic foils". Physics of Plasmas, 19 (8), 082710 (2012). doi:10.1063/1.4748579.

[32] S. I. Braginskii. Transport processes in a plasma, volume 1 of Reviews of Plasma Physics. Consultants Bureau, New York (1965). 